\title{
Mode I+III multiscale cohesive zone model: applications to facet orientation and toughening
}

\author{
V. Lazarus ${ }^{1}$, J.B. Leblond ${ }^{2}$, T. Cambonie ${ }^{3}$, B. Prabel $^{1}$ \\ ${ }^{1}$ IMSIA, ENSTA Paris, Institut Polytechnique de Paris, France \\ 2 Institut Jean Le Rond d'Alembert, Sorbonne Université, France \\ ${ }^{3}$ Laboratoire FAST, Université Paris-Saclay, CNRS, France \\ veronique.lazarus@ensta-paris.fr
}

\begin{abstract}
When subjected to some anti-plane shear mode III loading, segmentation of the crack front frequently occurs during propagation: even if the crack is initially planar, propagation produces facets/segments rotated toward the shear free direction [1]. These facets induce some modifications in the local loading of the crack tips that can be captured through a multi-scale cohesive zone model [2]: Assuming that the width of the facets is small in comparison to their length, the facets can be considered at the microscale, as a bidimensional periodic array of tilted cracks perpendicularly to the direction of propagation, and at the macroscale, as a growing Cohesive Zone. The model was developed initially supposing a constant period, small tilt angles and non-overlapping facets. We relaxed recently these assumptions to deal with more realistic cases including coarsening of the facets, large tilt angles and overlap. For this, the microscale problem is solved using XFEM and the outputs are further incorporated into the model to get some results on the macroscale, in particular the effective fracture energy. By comparing the results to some experiments [3], we demonstrate the ability of the approach (i) to determine the inclination of the facets and (ii) to quantify, in both fatigue and brittle fracture, the toughening due to the decrease of the crack opening driven by the unbroken ligaments between the facets.
\end{abstract}

\section{References}

[1] C.-H. Chen, T. Cambonie, V. Lazarus, M. Nicoli, A. J. Pons, and A. Karma. Crack Front Segmentation and Facet Coarsening in Mixed-Mode Fracture. Physical Review Letters, 115(26):265503, 2015.

[2] J.-B. Leblond, V. Lazarus, and A. Karma. Multiscale cohesive zone model for propagation of segmented crack fronts in mode I+III fracture. International Journal of Fracture (Special Invited Article Celebrating IJF at 50), 191(1):167-189, February 2015.

[3] V. Lazarus, B. Prabel, T. Cambonie, and J.B. Leblond. Mode I+III multiscale cohesive zone model with facet coarsening and overlap: Solutions and applications to facet orientation and toughening. Journal of the Mechanics and Physics of Solids, 141:104007, 2020. 